\title{
Large-amplitude acoustic solitary waves in a Yukawa chain
}

\author{
T. E. Sheridan ${ }^{1} \uparrow$ and James C. Gallagher ${ }^{1,2}$ \\ ${ }^{1}$ Department of Physics and Astronomy, Ohio Northern University, Ada, OH 45810, USA \\ ${ }^{2}$ Department of Physics, The Ohio State University, Columbus, OH 43210, USA
}

(Received 30 March 2017; revised 17 May 2017; accepted 18 May 2017)

We experimentally study the excitation and propagation of acoustic solitary waves in a one-dimensional dusty plasma (i.e. a Yukawa chain) with $n=65$ particles interacting through a screened Coulomb potential. The lattice constant $a=1.02 \pm 0.02 \mathrm{~mm}$. Waves are launched by applying a $100 \mathrm{~mW}$ laser pulse to one end of the chain for laser pulse durations from 0.10 to $2.0 \mathrm{~s}$. We observe damped solitary waves which propagate for distances $\gtrsim 30 a$ with an acoustic speed $c_{s}=11.5 \pm 0.2 \mathrm{~mm} \mathrm{~s}^{-1}$. The maximum velocity perturbation increases with laser pulse duration for durations $\leqslant 0.5 \mathrm{~s}$ and then saturates at $\approx 15 \%$. The wave speed is found to be independent of the maximum amplitude, indicating that the formation of nonlinear solitons is prevented by neutral-gas damping.

Key words: dusty plasmas, plasma nonlinear phenomena, plasma waves

\section{Introduction}

An acoustic soliton is a localized, supersonic, pulse-like excitation which propagates for long distances through a dispersive medium without dispersing because group velocity dispersion is balanced by nonlinear steepening. For small amplitudes $(\lesssim 10 \%)$, acoustic solitons are described by the nonlinear Korteweg de Vries (KdV) wave equation (Schmidt 1979; Nicholson 1983)

$$
\frac{\partial u}{\partial t}+A \frac{\partial^{3} u}{\partial \xi^{3}}+B \frac{\partial u^{2}}{\partial \xi}=0,
$$

where $t$ is a time variable, $\xi$ is a space variable in a frame moving with the acoustic speed $c_{s}$ and $u=u(\xi, t)$ is a field variable such as fluid velocity, density or potential. Here $A$ and $B$ are constants. The KdV equation admits both oscillatory solutions with speeds $\leqslant c_{s}$ (i.e. waves) and solitons with speeds $>c_{s}$. Exact solutions of the $\mathrm{KdV}$ equation can be found using the inverse scattering transform (Gardner et al. 1967), which shows any localized initial perturbation evolves into a continuum of dispersive waves and possibly one or more discrete solitons. The spatial profile of a KdV soliton is

$$
u(\xi)=\frac{3 \delta v}{c_{s}} \operatorname{sech}^{2}\left(\xi \sqrt{\frac{\delta v / c_{s}}{2}}\right),
$$

$\dagger$ Email address for correspondence: t-sheridan@onu.edu 
where the soliton speed in the laboratory frame $V=c_{s}+\delta v$. Consequently, the peak amplitude of a $\mathrm{KdV}$ soliton is directly proportional to $\delta v / c_{s}$, the soliton's normalized speed increment above the acoustic speed.

Melandsø (1996) has derived a KdV equation for weakly nonlinear, undamped waves in a one-dimensional (1-D) Yukawa chain. A Yukawa chain is a strongly coupled system of identical particles interacting through a Yukawa (i.e. Debye or screened Coulomb) potential. That is, the potential a distance $r$ from a particle with charge $q$ is given by

$$
V(r)=\frac{1}{4 \pi \epsilon_{0}} \frac{q}{r} \mathrm{e}^{-r / \lambda_{D}},
$$

where $\lambda_{D}$ is the Debye shielding length and $\epsilon_{0}$ is the permittivity of free space. Consequently, it is predicted that large-amplitude initial perturbations in a Yukawa chain will evolve into solitons for appropriate conditions. One signature of such solitons is an increase in speed with amplitude.

Dusty plasma, which is an experimental realization of the Yukawa chain, is made of nearly identical microscopic dust particles immersed in an electron-ion plasma. For typical laboratory plasma conditions, dust particles acquire a charge $q<0$. Large dust particles can be suspended above a horizontal electrode where the vertical electric force balances the particle's weight. In many cases, the potential well in the vertical direction is much deeper than that in the horizontal directions so the dusty plasma is two-dimensional. In the horizontal plane, each negatively charged dust particle is surrounded by a positive space charge cloud leading to Debye shielding. One-dimensional dusty plasmas (i.e. Yukawa chains) can be made by using additional confinement in the horizontal plane. Such configurations include straight lines in biharmonic wells (Homann et al. 1997; Liu, Avinash \& Goree 2003, 2004; Liu \& Goree 2005) and rings in annular wells (Sheridan 2009; Tkachenko, Sheridan \& Misko 2011; Sheridan \& Gallagher 2016).

A number of experiments have reported on linear wave propagation in 1-D dusty plasma. For both biharmonic (Homann et al. 1997; Misawa et al. 2001; Liu et al. 2003, 2004; Liu \& Goree 2005) and annular wells (Sheridan \& Gallagher 2016), small-amplitude longitudinal waves are found to be well described by the dispersion relation for a Yukawa chain (Melandsø 1996; Homann et al. 1997; Liu \& Goree 2005; Tkachenko et al. 2011; Sheridan \& Gallagher 2016), i.e. dust lattice waves. Chains confined in a biharmonic well can only hold a limited number of particles, restricting the distance over which waves can propagate before end effects become important (Liu \& Goree 2005). It is possible to create longer and more uniform chains using an annular well (Sheridan \& Gallagher 2016). The dispersion relation in such chains is well approximated (Sheridan 2009; Tkachenko et al. 2011) by the 1-D theory for straight line configurations when the ring diameter is much larger than the lattice constant, as verified experimentally (Sheridan \& Gallagher 2016). There have been several studies of the conditions under which nonlinear effects become important in 2-D dusty plasmas (Avinash et al. 2003; Nosenko et al. 2004; Sheridan, Nosenko \& Goree 2008). Experiments looking for nonlinear effects in 1-D dusty plasma chains are rarer.

In this paper, we describe an experiment where we launch an acoustic pulse in 1-D dusty plasma rings as a first step to creating solitons. We look for nonlinear effects, particularly an increase in wave speed with amplitude, which is a signature of nonlinear $\mathrm{KdV}$ behaviour. Section 2 gives the theory of linear dust lattice waves and $\S 3$ describes our experiment. Results and discussion are presented in $\S 4$ and conclusions are given in $\S 5$. 


\section{Dust lattice waves}

The dispersion relation for undamped linear dust lattice waves (DLW) including nearest and next-nearest neighbour interactions is (Melandsø 1996; Tkachenko et al. 2011)

$$
\frac{\omega_{l}^{2}}{\omega_{0}^{2}}=\mathrm{e}^{-\bar{\kappa}}\left(1+\bar{\kappa}+\frac{\bar{\kappa}^{2}}{2}\right) \sin ^{2} \frac{k a}{2}+\frac{\mathrm{e}^{-2 \bar{\kappa}}}{8}\left(1+2 \bar{\kappa}+2 \bar{\kappa}^{2}\right) \sin ^{2} k a
$$

where $\omega_{l}$ is the (longitudinal) wave frequency, $k$ is the wavenumber and $a$ is the lattice constant. Frequency is normalized by

$$
\omega_{0}^{2}=\frac{8 q^{2}}{4 \pi \epsilon_{0} m a^{3}},
$$

which is the dust lattice oscillation frequency for a Coulomb chain. Here $q$ is the particle charge and $m$ is the mass. The normalized dispersion relation depends only on the Debye screening parameter

$$
\bar{\kappa}=\frac{a}{\lambda_{D}},
$$

where $\bar{\kappa}=0$ for an unshielded Coulomb interaction.

As shown in figure 1(a), the DLW dispersion is an acoustic one. The acoustic speed $(k a \ll 1)$ for dust lattice waves can be written as

$$
C_{D L W}=\sqrt{\frac{3 q^{2}}{4 \pi \epsilon_{0} m a}} f(\bar{\kappa}),
$$

with

$$
f(\bar{\kappa})=\sqrt{\frac{2}{3} \mathrm{e}^{-\bar{\kappa}}\left(1+\bar{\kappa}+\frac{1}{2} \bar{\kappa}^{2}\right)+\frac{1}{3} \mathrm{e}^{-2 \bar{\kappa}}\left(1+2 \bar{\kappa}+2 \bar{\kappa}^{2}\right)} .
$$

The square root term in (2.4) gives the acoustic speed for an unshielded Coulomb interaction, while $f(\bar{\kappa})$ gives the reduction in the normalized acoustic speed due to increased Debye shielding. The dependence of the normalized acoustic speed (i.e. $f(\bar{\kappa})$ ) on $\bar{\kappa}$ is shown in figure $1(b)$, from which we see that the acoustic speed has only a weak dependence on $\bar{\kappa}$ when $\bar{\kappa} \lesssim 1$, which is often the case in 1-D dusty plasma experiments (Sheridan \& Gallagher 2016). The real part of the dispersion relation is only slightly modified by weak damping $\gamma \ll \omega_{0}$. For the experiment described here, damping $\gamma / \omega_{0}=0.06 \ll 1$ is weak.

Consequently, for a chain of identical particles, the properties of linear dust lattice waves only depend on two dimensionless parameters: the screening parameter $\bar{\kappa}$ and the normalized damping rate $\gamma / \omega_{0}$. KdV theory tells us that the properties and evolution of nonlinear (i.e. large amplitude) waves also depend on the peak wave amplitude. In particular, there may be threshold behaviour for the creation of solitons such that subthreshold perturbations are damped (Avinash et al. 2003) while above threshold perturbations evolve into solitons whose speed increases with peak amplitude. 

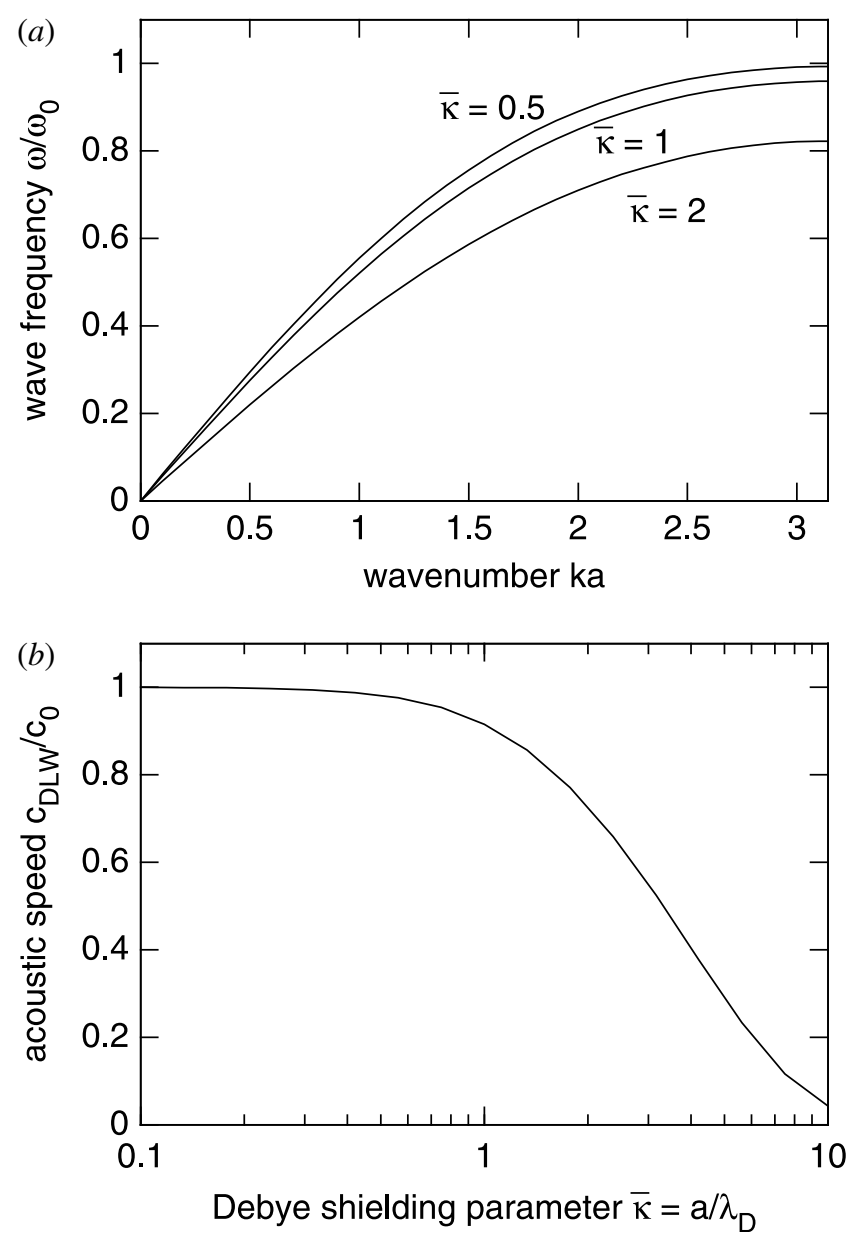

FIGURE 1. (a) Dispersion relations for acoustic waves in a 1-D dusty plasma lattice for the Debye screening parameter $\bar{\kappa}=0.5,1$ and 2. (b) A plot of acoustic speed versus $\bar{\kappa}$ shows that the acoustic speed does not depend strongly on $\bar{\kappa}$ when $\bar{\kappa} \lesssim 1$.

\section{Experiment}

We have studied acoustic solitary wave propagation in a 1-D dusty plasma lattice in the DONUT (Dusty Ohio Northern University experimenT) experiment. An $89 \mathrm{~mm}$ diam. aluminium electrode (figure 2) was placed in the vacuum chamber. An annular groove in the electrode $(16.0 \mathrm{~mm}$ inner diameter $\times 40.0 \mathrm{~mm}$ outer diameter $\times 6.3 \mathrm{~mm}$ deep) created a potential well that confines dust particles in a 1-D ring (Sheridan \& Gallagher 2016). An additional aluminium ring $(89 \mathrm{~mm}$ outer diameter $\times 75.7 \mathrm{~mm}$ inner diameter $\times 6.0 \mathrm{~mm}$ deep) was placed on the outside of the electrode to deepen the transverse potential well to prevent transverse buckling of the chain when it is compressed. Argon was bled into the vacuum vessel while the vacuum pump was throttled, and the pressure was stabilized at 13.4 mTorr. Plasma was generated by applying $\approx 3.5 \mathrm{~W}$ of radio-frequency (r.f.) power at $13.56 \mathrm{MHz}$ to the electrode. On the electrode, the peak-to-peak r.f. voltage was $112 \mathrm{~V}$ and the direct current self-bias was $-53 \mathrm{~V}$.

Melamine-formaldehyde (MF) spheres with a diameter $8.94 \pm 0.09 \mu \mathrm{m}$ (Sheridan 2005) and a mass $m=5.65 \times 10^{-13} \mathrm{~kg}$, were trapped in the annular potential well. 


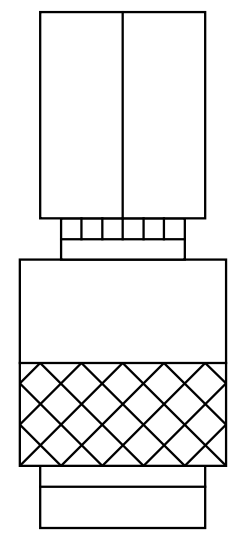

$1 / 2$ in. CCD

video camera

$0.75 x$ adapter

$55 \mathrm{~mm} \mathrm{f} / 2.8$ telecentric lens

red filter

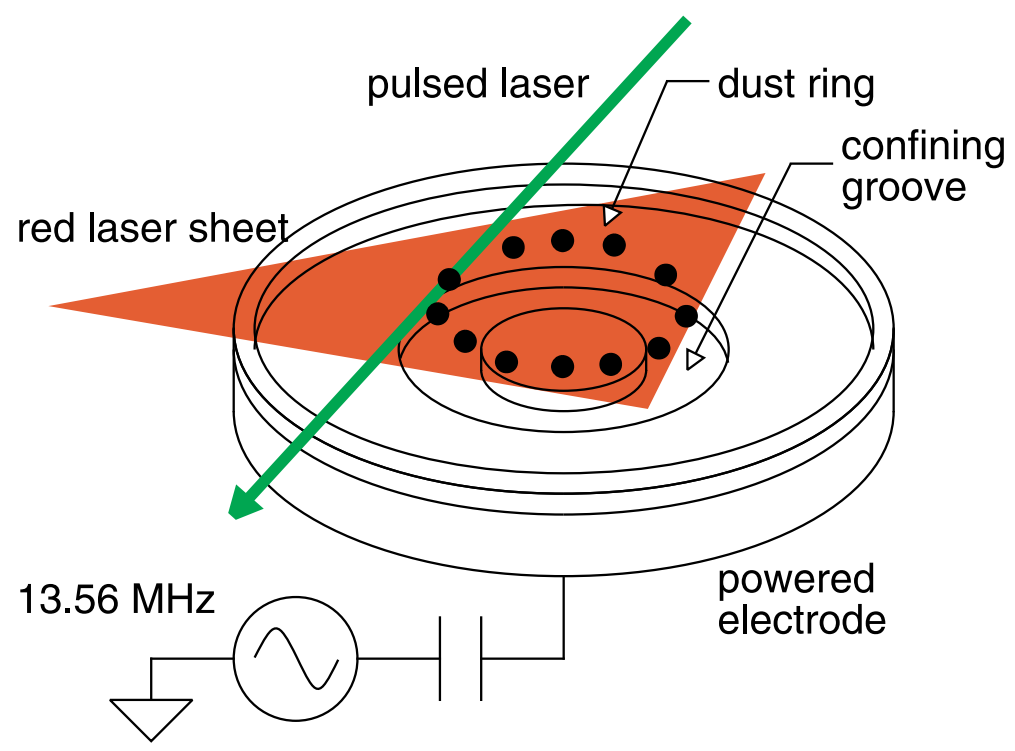

FIGURE 2. Schematic of the experimental set-up. An incomplete dust ring with $n=65$ particles is levitated above the electrode. Pulses from a green diode laser compress the end of the chain and launch large-amplitude acoustic waves. Particles are illuminated using a red laser and their motion is recorded using the video camera.

The particles were illuminated using a sheet of red laser light and their positions were recorded using a top view video camera. The camera resolution was $1024 \times$ 1024 pixels, where one pixel corresponds to $30.76 \times 30.76 \mu \mathrm{m}^{2}$ at the dusty plasma ring. Movies of 1024 frames at 15 frames $\mathrm{s}^{-1}$ were recorded for later analysis.

An incomplete ring was formed with $n=65$ particles, as shown in figure $3(a)$. The radius of the ring was $11.58 \mathrm{~mm}$, and the particles were levitated $9.7 \mathrm{~mm}$ above the top of the outer confining ring. The mean lattice spacing $a=1.02 \pm 0.02 \mathrm{~mm}$ (figure $3(b)$ ) exhibits a modulation due to small variations in the depth of the well. The dependence of these local minima depend on the r.f. power and matching network settings, indicating they are associated with a small standing wave component in the r.f. electric field. The neutral gas damping rate was $\gamma=1.3 \mathrm{~s}^{-1}$ (Sheridan 2005). 

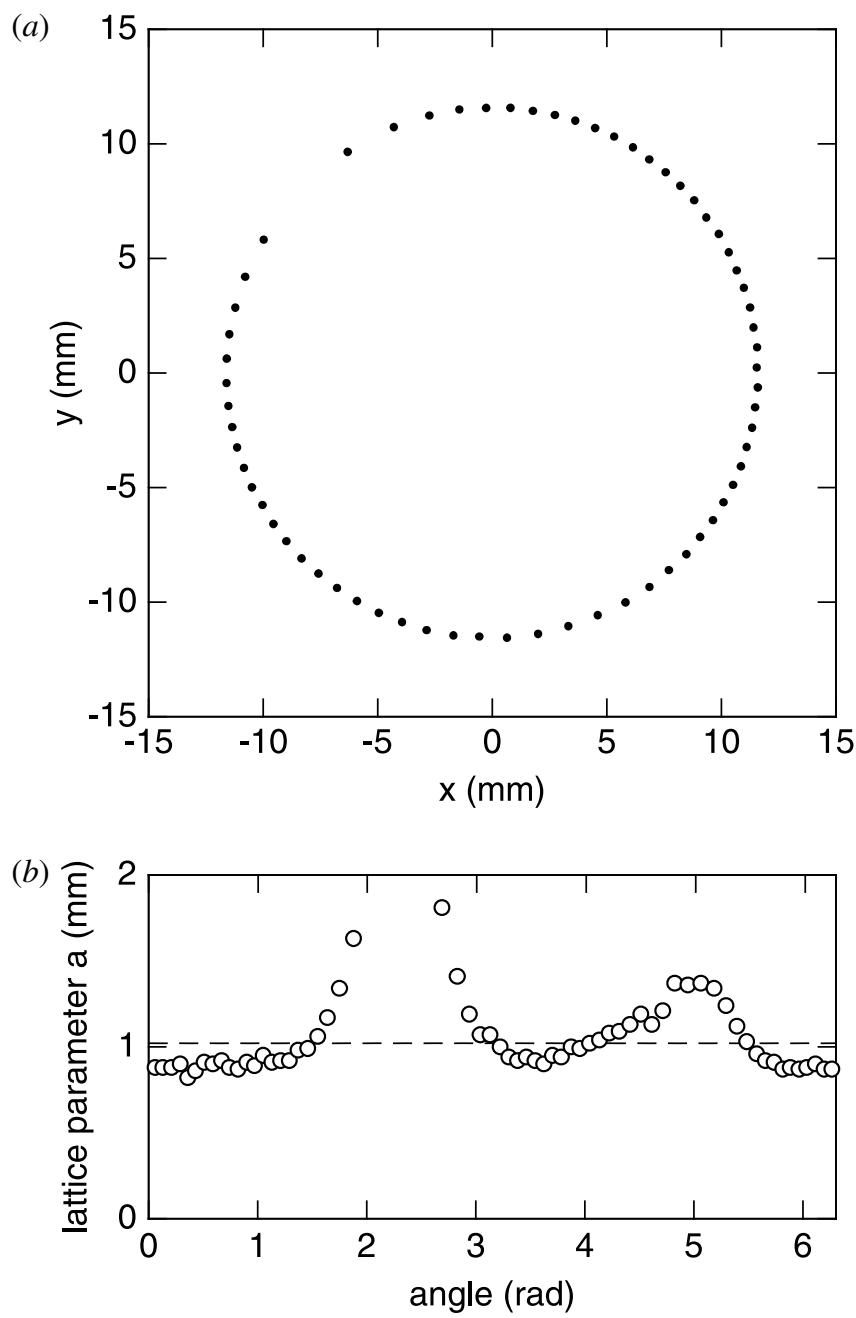

Figure 3. (a) Positions of $n=65$ dust particles. The dust ring has a gap in the upper left corner, which prevents a counter-propagating wave from being launched when the excitation laser strikes the particles. The radius of this ring is $11.58 \mathrm{~mm}$. (b) The lattice space is plotted as a function of particle position. Excluding the gap region, the average lattice constant is $a=1.02 \pm 0.02 \mathrm{~mm}$ is shown with the broken line.

From similar experiments in DONUT using the same dust particles $q \approx-1.2 \times 10^{4} e$ (Sheridan \& Gallagher 2016). From (2.2), the unshielded longitudinal oscillation frequency $\omega_{0} \approx 21 \mathrm{rad} \mathrm{s}^{-1}$, giving a normalized damping rate $\gamma / \omega_{0}=0.06$.

Longitudinal waves were launched by compressing the end of the chain using a $100 \mathrm{~mW}$ (nominal), $532 \mathrm{~nm}$ diode laser which was pulsed on for a duration $\Delta t_{\text {laser }}$, with $10.0 \mathrm{~s}$ between laser pulses. The beam struck several particles at one end of a gap in the dust ring causing a wave to propagate counter-clockwise around the ring. The gap in the dust ring (figure $3(a)$ ) prevented a rarefactive pulse from propagating in the opposite direction and interfering with the compressive pulse. To look for a nonlinear increase of pulse speed with wave amplitude, we increased the maximum perturbation by increasing laser on-time $\Delta t_{\text {laser }}$ from 0.1 to $2.0 \mathrm{~s}$ with values of 0.1 , 


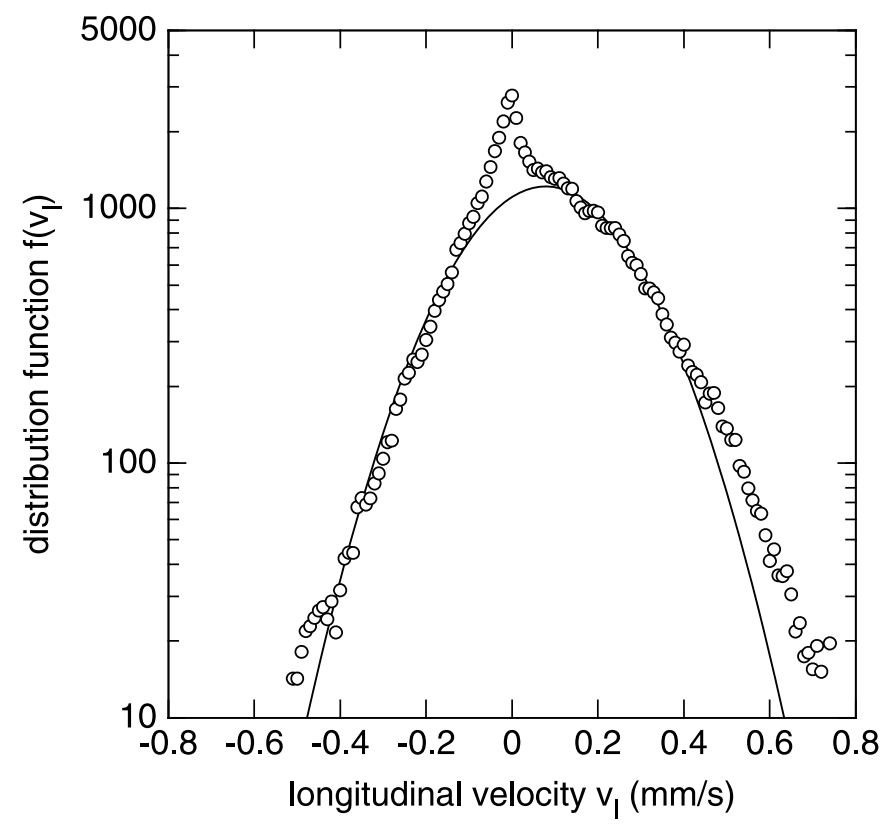

FIGURE 4. The longitudinal velocity distribution function for the unperturbed dust ring. The distribution function is well fitted by a drifting Maxwellian distribution with a kinetic temperature $T=1300 \mathrm{~K}$.

$0.2,0.3,0.5,0.7,1.0,1.4$ and $2.0 \mathrm{~s}$. Data for $\Delta t_{\text {laser }}=1.0 \mathrm{~s}$ were not usable because the illumination laser was misaligned.

\section{Results and discussion}

The longitudinal velocity distribution function for dust particles in the unperturbed ring is shown in figure 4 . Velocities in the interval $v \approx 0.0 \pm 0.15 \mathrm{~mm} \mathrm{~s}^{-1}$ cannot be resolved due to the camera's finite pixel size. The velocity distribution function is well fitted by a drifting Maxwellian. The drift velocity of $0.078 \mathrm{~mm} \mathrm{~s}^{-1}$ represents a slow counter-clockwise rotation of the ring which is much less than the dust lattice wave speed. This rotation may be due to neutral-gas flow in the chamber (Carstensen, Greiner \& Piel 2010). The kinetic temperature of the fitted curve is $1300 \mathrm{~K}$. The temperature of stationary rings has previously been measured as $\approx 400 \mathrm{~K}$ (Sheridan \& Gallagher 2016), indicating that dust lattice is slightly heated due to the conversion of rotational motion into random kinetic energy. The lattice is strongly coupled. From the thermally excited radial breathing mode (Sheridan \& Gallagher 2016), we find the transverse (radial) oscillation frequency is $\omega_{t 0}=22.3 \pm 0.3 \mathrm{rad} \mathrm{s}^{-1}$, which gives the depth of the radial potential well.

To characterize acoustic pulse propagation, we created space-time plots of the longitudinal (azimuthal) particle velocity. The space-time plot for $\Delta t_{\text {laser }}=1.4 \mathrm{~s}$ is shown in figure 5. From these space-time plots, we determined the group velocity of the acoustic pulse. For all values of $\Delta t_{\text {laser }}$, the large-amplitude acoustic wave has a speed comparable to the background wave noise. The background waves, which propagate at the acoustic speed, are associated with lattice relaxation as the dust ring rotates into non-equilibrium configurations, and so propagate for long distances. 


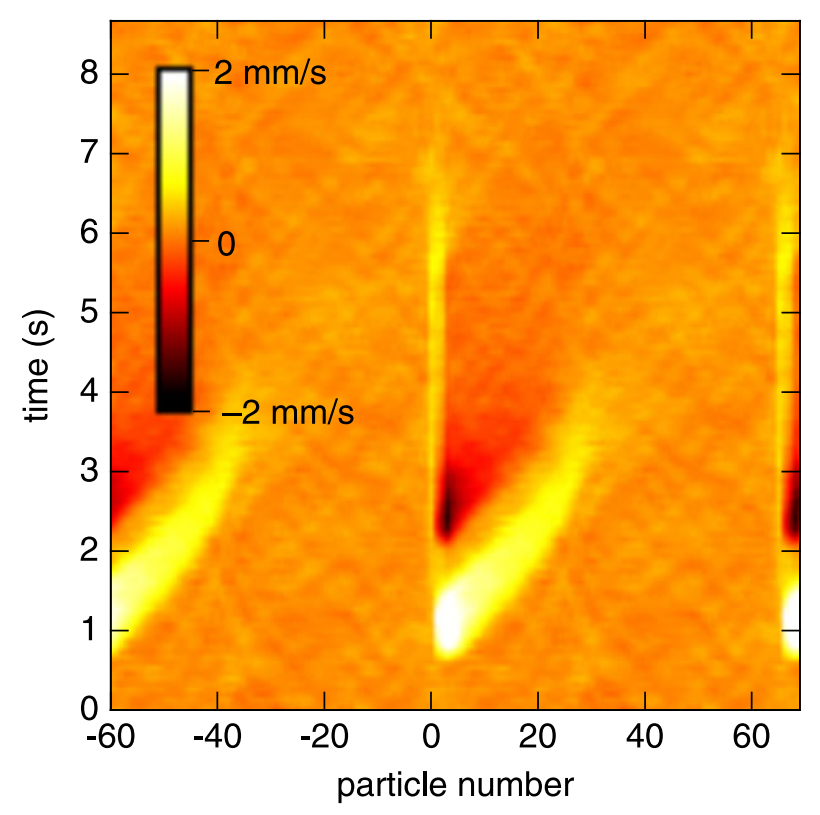

FIGURE 5. Space-time plot of longitudinal particle velocities for a laser pulse duration $\Delta t_{\text {laser }}=1.4 \mathrm{~s}$. We plot two periods around the ring. A compressive acoustic pulse is excited by the laser pulse and a dispersive rarefactive pulse forms when the laser is switched off. Background acoustic noise is evident with a velocity close to the laser-launched pulse.

For $\Delta t_{\text {laser }} \gtrsim 0.5 \mathrm{~s}$, a rarefactive wave was launched when the laser was turned off, and also travelled at the acoustic speed. We observed large-amplitude acoustic pulses with a velocity perturbation amplitude $\approx 10 \%$ of the acoustic speed which propagate for a significant distance. If this solitary wave was described by $\mathrm{KdV}$ theory we would expect it to have a speed $\approx 1.03 C_{D L W}$. However, it appears that neutral-gas damping dominates nonlinear effects and the pulse does not evolve into a soliton. Interestingly, the damping does not appear to be linear - rather the leading edge of the pulse slows below the acoustic speed and the entire pulse is extinguished. This is consistent with nonlinear steepening moving wave energy to higher wavenumbers with lower group velocities.

One measure of the wave amplitude is with the maximum velocity perturbation $\delta v_{\max }$ for particles in the chain. In figure $6(a)$ we plot $\delta v_{\max }$ for particle numbers $i=7$, 12 and 17 , where $i=1$ is the first particle struck by the laser beam versus laser pulse duration $\Delta t_{\text {laser }}$. Starting with $\Delta t_{\text {laser }}=0.1 \mathrm{~s}$ we find that the wave amplitude increases for $\Delta t_{\text {laser }} \leqslant 0.5 \mathrm{~s}$ and then saturates for $\Delta t_{\text {laser }}>0.5 \mathrm{~s}$. This saturation occurs because after the laser is on for some time the dust particles are pushed into new equilibrium positions; thus the greater impulse imposed by the laser is counteracted by the lattice restoring force. In these cases, a rarefactive wave is launched after the laser is turned off as can be seen in figure 5. These curves give a spatial damping coefficient $k_{i}=$ $0.07 \mathrm{~mm}^{-1}$, which is consistent with neutral-gas (Epstein drag) damping.

The velocity of the solitary waves versus laser pulse duration is plotted in figure $6(b)$. For $\mathrm{KdV}$-like solitons we expect the velocity to increase as the velocity perturbation (wave amplitude) increases, as predicted by KdV theory (Schmidt 1979) and as observed in 2-D dusty plasma (Sheridan et al. 2008). This is not what we 


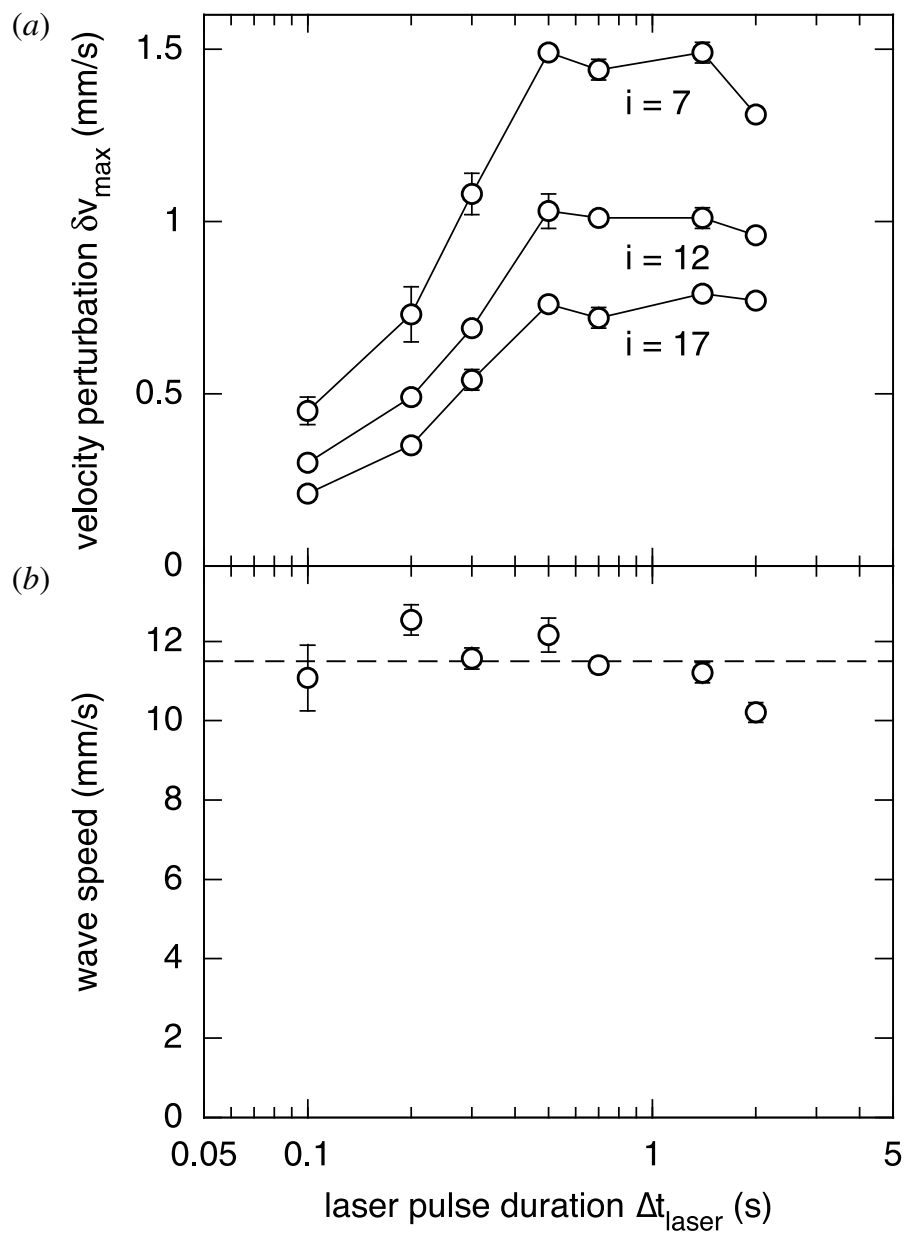

FIGURE 6. (a) Maximum velocity perturbation $\delta v_{\max }$ for particle numbers $i=7,12$ and 17 versus laser pulse duration. Spatial damping is evident. At all three particle positions, the amplitude increases with laser pulse duration until the amplitude saturates for $\Delta t_{\text {laser }} \gtrsim 0.7 \mathrm{~s}$. $(b)$ Measured speed of the acoustic pulse versus $\Delta t_{\text {laser }}$. The wave speed is effectively independent of $\Delta t_{\text {laser }}$, and therefore the wave amplitude, indicating that the wave propagation is linear. The average acoustic speed of $11.5 \pm 0.2 \mathrm{~mm} \mathrm{~s}^{-1}$ is shown by the broken line.

observe. We find that the wave speed is independent of the maximum velocity perturbation, both when the pulse amplitude is increasing for $\Delta t_{\text {laser }} \leqslant 0.5 \mathrm{~s}$ and when then pulse amplitude is saturated at $\delta v_{\max } / C_{D L W} \gtrsim 15 \%$. That is, nonlinear effects do not overcome damping in this experiment. The average dust lattice wave speed is $C_{D L W}=11.5 \pm 0.2 \mathrm{~mm} \mathrm{~s}^{-1}$. If we take $\bar{\kappa}=1$ (Sheridan \& Gallagher 2016) as a typical value, then from (2.4), $q=-(11500 \pm 500) e$, which is consistent with previous measurements (Sheridan 2005; Sheridan \& Gallagher 2016).

\section{Conclusions}

We have studied the propagation of large-amplitude acoustic pulse-like waves in a long 1-D dusty plasma (i.e. a Yukawa chain). As a first step toward creating solitons, 
we used laser pulses of increasing duration to create initial velocity perturbations. The wave amplitude increases with laser pulse duration for $\Delta t_{\text {laser }} \leqslant 0.5 \mathrm{~s}$, and then saturates when the laser force is balanced by the lattice restoring force. Waves with maximum perturbation amplitudes of $\approx 15 \%$ were launched, and propagated as unipolar longitudinal compressions for $\gtrsim 30$ lattice constants. The wave speed is independent of the maximum velocity perturbation, indicating that the pulses do not evolve into nonlinear (KdV) solitons. Damping, $\gamma / \omega_{0} \approx 0.06$, dominates the nonlinear effects so that the pulses decay. The decay mechanism does not appear to be simple linear damping. Rather, the leading edge of the pulse slows to below the acoustic speed, which may indicate nonlinear steepening. Creating nonlinear solitary waves in this system will require either reduced damping, larger perturbations amplitudes, or both.

\section{REFERENCES}

Avinash, K., Zhu, P., Nosenko, V. \& Goree, J. 2003 Nonlinear compressional waves in a two-dimensional Yukawa lattice. Phys. Rev. E 68 (4), 046402.

Carstensen, J., Greiner, F. \& Piel, A. 2010 Determination of dust grain charge and screening lengths in the plasma sheath by means of a controlled cluster rotation. Phys. Plasmas 17 (8), 083703 .

Gardner, C. S., Greene, J. M., Kruskal, M. D. \& Miura, R. M. 1967 Method for solving Korteweg-deVries equation. Phys. Rev. Lett. 19 (19), 1095-1097.

Homann, A., Melzer, A., Peters, S. \& Piel, A. 1997 Determination of the dust screening length by laser-excited lattice waves. Phys. Rev. E 56 (6), 7138-7141.

LiU, B., Avinash, K. \& Goree, J. 2003 Transverse optical mode in a one-dimensional Yukawa chain. Phys. Rev. Lett. 91 (25), 255003.

LiU, B., Avinash, K. \& Goree, J. 2004 Characterizing potentials using the structure of a onedimensional chain demonstrated using a dusty plasma crystal. Phys. Rev. E 69 (3), 036410.

LiU, B. \& Goree, J. 2005 Phonons in a one-dimensional Yukawa chain: dusty plasma experiment and model. Phys. Rev. E 71 (4), 046410.

Melandsø, F. 1996 Lattice waves in dust plasma crystals. Phys. Plasmas 3 (11), 3890-3901.

Misawa, T., Ohno, N., Asano, K., Sawai, M., Takamura, S. \& Kaw, P. K. 2001 Experimental observation of vertically polarized transverse dust-lattice wave propagating in a one-dimensional strongly coupled dust chain. Phys. Rev. Lett. 86 (7), 1219-1222.

Nicholson, D. R. 1983 Introduction to Plasma Theory. Wiley.

Nosenko, V., Avinash, K., Goree, J. \& LiU, B. 2004 Nonlinear interaction of compressional waves in a 2D dusty plasma crystal. Phys. Rev. Lett. 92 (8), 085001.

SCHMidt, G. 1979 Physics of High Temperature Plasmas. Academic.

SHERIDAN, T. E. 2005 Center-of-mass and breathing oscillations in small complex plasma disks. Phys. Rev. E 72 (2), 026405.

Sheridan, T. E. 2009 Dusty plasma ring model. Phys. Scr. 80 (6), 065502.

Sheridan, T. E. \& Gallagher, J. C. 2016 Dusty plasma (Yukawa) rings. Phys. Plasmas 23 (11), 110703.

Sheridan, T. E., Nosenko, V. \& Goree, J. 2008 Experimental study of nonlinear solitary waves in two-dimensional dusty plasma. Phys. Plasmas 15 (7), 073703.

Tkachenko, D. V., Sheridan, T. E. \& Misko, V. R. 2011 Dispersion relations for circular single and double dusty plasma chains. Phys. Plasmas 18 (10), 103709. 